\title{
Evaluation of sulphadoxine-pyrimethamine for intermittent preventive treatment of malaria in pregnancy: a retrospective birth outcomes study in Mansa, Zambia
}

Kimberly E Mace ${ }^{1 *}$, Victor Chalwe ${ }^{2}$, Bonnie L Katalenich ${ }^{3}$, Michael Nambozi ${ }^{4}$, Luamba Mubikayi ${ }^{5}$, Chikuli K Mulele $^{6}$, Ryan E Wiegand', Scott J Fillerr, Mulakwa Kamuliwo ${ }^{7}$, Allen S Craig ${ }^{1}$ and Kathrine R Tan ${ }^{1}$

\begin{abstract}
Background: Intermittent preventive treatment of malaria in pregnancy (IPTP) with sulphadoxine-pyrimethamine (SP) decreases placental parasitaemia, thus improving birth outcomes. Zambian policy recommends monthly SP-IPTp doses given presumptively during pregnancy at each antenatal examination, spaced one month apart after 16 weeks of gestation. The effectiveness of SP-IPTp was evaluated in Zambia where a recent study showed moderate prevalence of Plasmodium falciparum parasites with genetic mutations that confer SP resistance.
\end{abstract}

Methods: HIV-negative women were enrolled at the time of delivery at two facilities in Mansa, Zambia, an area of high malaria transmission. Women were interviewed and SP exposure was determined by antenatal card documentation or self-reports. Using Poisson regression modelling, the effectiveness of SP-IPTp was evaluated for outcomes of parasitaemia (microscopic examination of maternal peripheral, cord, and placental blood films), maternal anaemia ( $\mathrm{Hb}<11 \mathrm{~g} / \mathrm{dl})$, placental infection (histopathology), and infant outcomes (low birth weight (LBW), preterm delivery, and small for gestational age) in women who took 0-4 doses of SP-IPTp.

Results: Participants included 435 women, with a median age of 23 years (range 16-44). Thirty-four women took zero doses of SP-IPTp, while 115, 142 and 144 women took one, two, or $\geq$ three doses, respectively. Multivariate Poisson regression models considering age, mosquito net usage, indoor residual spraying, urban home, gravidity, facility, wet season delivery, and marital status showed that among paucigravid women $\geq$ two doses of SP-ITPp compared to one or less doses was associated with a protective effect on LBW (prevalence ratio (PR) 0.33, 95\% confidence interval (Cl) 0.12-0.91) and any infection (PR 0.76, Cl 0.58-0.99). Multivariate models considering SP-IPTp as a continuous variable showed a protective dose-response association with LBW (paucigravid women: PR 0.54, Cl 0.33-0.90, multigravid women: PR 0.63, Cl 0.41-0.97).

Conclusions: In Mansa, Zambia, an area of moderate SP resistance, $\geq$ two doses of SP-IPTp were associated with a protective effect from malaria in pregnancy, especially among paucigravid women. Each dose of SP-IPTp contributed to a 46 and 37\% decrease in the frequency of LBW among paucigravid and multigravid women, respectively. SP-IPTp remains a viable strategy in this context.

Keyword: Plasmodium falciparum malaria, Intermittent preventive treatment of malaria in pregnancy, Sulphadoxinepyrimethamine, Zambia

\footnotetext{
*Correspondence: kmace@cdc.gov

${ }^{1}$ Malaria Branch, Division of Parasitic Diseases and Malaria, Center for Global

Health, Centers for Disease Control and Prevention, Atlanta, GA, USA

Full list of author information is available at the end of the article
} 


\section{Background}

Malaria during pregnancy is dangerous for women and infants. Approximately 32 million pregnancies per year occurring in regions of sub-Saharan Africa are at risk for malaria [1], and an estimated 200,000 of them occur in Zambia [2]. Malaria infection during pregnancy can result in adverse effects for both mother and infant, including severe maternal anaemia, low birth weight (LBW), and increased perinatal and infant mortality; adverse effects are more likely during a woman's first or second pregnancy [3]. Women infected with HIV have a higher risk for adverse pregnancy outcomes [4], and the beneficial effects of SP-IPTp can be compromised by HIV status. Malaria is a substantial health concern in Zambia. In 2010, at the time of the study, the national prevalence of malaria parasitaemia in children under five years old was $16 \%$; Luapula Province had the highest prevalence with 51\% of children under five years infected [5]. The Zambian Ministry of Health recommends preventive measures to protect pregnant women from malaria including sleeping under an insecticide-treated mosquito net (ITN), and taking sulphadoxine-pyrimethamine (SP) regardless of malaria parasitaemia, after 16 weeks of gestation as intermittent preventive treatment in pregnancy (IPTp) with SP (SP-IPTP) [6]. At the time of the study, the Zambia guidelines recommended three doses of SP-IPTp, spaced one month apart in the second and third trimesters of pregnancy. Subsequently, Zambian guidelines were updated to be in line with the WHO 2012 recommendations for monthly dosing of SP-IPTp given at each antenatal visit in the second and third trimesters [7].

SP-IPTp protects from malaria during pregnancy, likely by clearing existing asymptomatic infections and by preventing new infections from occurring during the period after treatment, due to SP's long half-life [8]. Earlier studies showed that SP-IPTp decreased placental malaria parasitaemia [9-17], maternal anaemia [11,12,15,18], and LBW $[15-17,19]$. Parasite resistance to SP threatens the effectiveness of SP-IPTp. Indeed malaria parasites developed SP resistance in Zambia [20] and in 2006 the adequate clinical and parasitological response for SP in children in Zambia was only 77\% [21]. Other studies in sub-Saharan Africa suggest that SP-IPTp effectiveness is weakening $[22,23]$. The presence of Plasmodium falciparum dihydropteroate synthase (dhps) and dihydrofolate reductase (dhfr) mutations are associated with SP drug resistance in the Plasmodium parasite. The quintuple mutant haplotype, consisting of the N51I, C59R, S108N substitutions in $d h f r$ and the $\mathrm{A} 437 \mathrm{G}$ and $\mathrm{K} 540 \mathrm{E}$ substitutions in dhps, has been associated with SP treatment failure [24]. A study conducted concurrently to the present study in Mansa, Zambia showed that among 84 specimens with complete haplotypes at $d h f r$ loci 51, 59 and 108, and dhps loci 437 and 540, 61\% had the quintuple mutant [25]. In comparison to other malaria regions, Zambia has intermediate frequencies of mutations that cause resistance to SP. A study conducted in Zambia showed that HIV-infected women require monthly dosing to obtain the benefits of SP-IPTp [26], however there is no comparable study for HIV-negative women in Zambia.

This study was designed in collaboration with the Malaria in Pregnancy (MiP) Consortium as part of a multi-site investigation into the effectiveness of SP-IPTp, where Zambia is one of eight sites in six countries, including Mali (two sites), Burkina Faso, Uganda, Kenya, and Malawi (two sites). Three modules were designed, including a therapeutic efficacy evaluation, determination of molecular markers of SP resistance and a retrospective cohort assessment of birth outcomes. This manuscript describes the Zambia birth outcomes component; the compiled analysis from the multi-site analysis and results from the other modules and sites are reported separately [25,27-30].

\section{Methods}

\section{Study design and population}

In Zambia, the retrospective-cohort birth outcomes study was conducted in Mansa, Luapula Province, at the Mansa General Hospital and Senama Rural Health Clinic. Both facilities serve the Mansa District population; Mansa General Hospital is a level-2 referral hospital with 326 beds and is located $0.5 \mathrm{~km}$ from the Mansa District Health Management Office (DHMO), and Senama Rural Health Clinic is situated $3 \mathrm{~km}$ from the DHMO, outside Mansa urban centre and has 20 beds [31]. The study period was from December 2009 to December 2010, spanning portions of the 2009-2010 and 2010-2011 rainy seasons, occurring generally from November to April. Women who presented for delivery at the participating facilities were enrolled by midwives trained in sample collection procedures related to this study. Inclusion criteria were: having an available SP-IPTp and HIV-test history recorded on an antenatal card, a singleton delivery, being $\geq 16$ years old, and providing informed consent. Since HIV-positive women are recommended to take cotrimoxazole prophylaxis to prevent opportunistic infections, and SP-IPTp is not advised for pregnant women on co-trimoxazole due to toxicity concerns from simultaneous administration of the two drugs with similar mechanisms of action [8], only HIV-negative women were enrolled in this study. Women were excluded if they had ever had a positive HIV test result, if after the 16th gestational week they received either a blood transfusion or an anti-malarial medication other than SP-IPTp, if they delivered outside of the hospital, or if they lived outside the Mansa District. Despite the requirement to enrol only women who had an antenatal card, because stock-outs of SP occurred during the study period, it was possible to enrol women who did not take any SP-IPTp doses. 
Sometimes eligible women who birthed at the two facilities could not be screened and enrolled because a study-trained midwife was not on duty, or could not accommodate specimen and data collection procedures in addition to routine hospital responsibilities, or when study materials were stocked out. To minimize the likelihood of systematic selection bias, the study-trained midwives included those of both supervisor and non-supervisor status, and also included those that worked variable shifts, including during the day and night time.

\section{Parameters evaluated and outcome definitions}

Enrolled women were interviewed using MiP Consortiumdesigned data collection instruments for the multi-site study, modified specifically for the Zambian context. Information collected included: demographics, ITN ownership and use, indoor residual spraying (IRS), and medical history. Antenatal cards served as the primary source of documented SP-IPTp dates. However, a small number of self-reported SP-doses, which were commonly associated with SP stock-outs at the antenatal clinics, were accepted. When possible, these doses were verified with receipts from purchase at pharmacies or from other medical records because women often filled the SP-IPTp prescription and retained the receipt with her ANC card. During data analysis, the dates of the non-antenatal clinic SP doses were validated to ensure they were not duplicated with those reported on the antenatal card. For all enrolled women, the study team of nurse midwives attempted to collect the following soon after delivery: haemoglobin $(\mathrm{Hb})$ measurements, blood smears from maternal peripheral, placental and cord blood, placental specimens for histopathology, infant birth weight, gestational age at delivery assessed by Ballard score [32], and last menstrual period (LMP). Babies with a birth weight of $\leq 2,500 \mathrm{~g}$ were followed up at six weeks to assess mortality.

Moderate maternal anaemia was defined as $\mathrm{Hb}<11 \mathrm{~g} / \mathrm{dl}$; severe maternal anaemia was $\mathrm{Hb}<8 \mathrm{~g} / \mathrm{dl}$. Placental infection according to histopathology was classified according to the following guidelines: class 1: parasites present with no pigment in monocytes or fibrin; class 2: parasites present with pigment in monocytes +/- fibrin; class 3: parasites present with pigment in fibrin; class 4 : no parasites, pigment only indicating past infection; class 5 : no parasites or pigment present, indicating no infection [33]. Maternal, cord and placental blood smears were examined for presence of malaria parasites, plus parasite species and density for those positive. Infants weighing $\leq 2,500 \mathrm{~g}$ at birth were considered to be LBW; small for gestational age (SGA) was defined as being less than the tenth percentile in weight per gestational age determined by a longitudinal ultrasound-derived foetal nomogram for a sub-Saharan African population [34]. Preterm was defined as birth at $<37$ weeks of completed gestation.
Finally, a 'composite poor birth outcome' was defined as any infant born with LBW, SGA or preterm.

\section{Laboratory methods \\ Malaria blood smears}

Thick and thin smears were prepared from finger-stick blood samples following a standard protocol using a 5\% Giemsa solution [35]. Trained, on-site technicians read thick blood smears to detect Plasmodium parasites and to calculate the parasite density according to the number of asexual parasites per 300 white blood cells (WBCs), assuming a total of 8,000 WBCs per $\mu$ l. A thick blood smear was classified as negative if no asexual parasites were identified after 1,000 WBCs were counted. Thin blood smears were examined to determine Plasmodium species. For quality control, all slides were sent to a national-level laboratory and read a second time by a senior microscopist. If the results of the two readings were discrepant then the slide was read for a third time by a national-level, senior microscopist for a final determination.

Upon delivery, samples of placenta and placental blood were collected. An incision was made on the maternal side of the placenta and pooled blood was collected for thick smear examination as described for malaria blood smears.

\section{Placental histopathology}

Tissue samples from the maternal side of the placenta were collected; dimensions were approximately $2 \mathrm{~cm} \times$ $2 \mathrm{~cm}$ in length and width, and $1 \mathrm{~cm}$ in depth. Specimens were immediately placed in buffered formalin-filled bottles and stored at room temperature. Within one month of collection, specimens were sent to Tropical Disease Research Centre (TDRC), Ndola, Zambia where tissues were wax embedded within two weeks, sectioned, stained with Gurr's modified Giemsa or haematoxylin and eosin, and examined independently by two senior pathologists experienced in reading placental histopathology for malaria. For discrepant results, specimens were reviewed and consensus was determined. Placental histology was classified on a five-point scale as described by Rogerson et al. [33] and also characterized as active placental infection (class 1), chronic placental infections (classes 2 and 3), and past malaria placental infection (class 4 ).

\section{Sample size}

Sample size was calculated for the main birth outcome of interest, evidence of placental malaria by histopathology. The major strata of interest for the primary independent variable, SP-IPTp doses, was 'two or more doses of SP' (reflecting full uptake of the WHO IPTp recommendations) versus 'one or less doses of SP'; based on the 2008 Zambian National Malaria Indicator Survey, 66\% of women surveyed received at least two doses of SP-IPTp [36]. It was also estimated that $20 \%$ of untreated women 
would have placental malaria and that treatment with at least two doses of SP-IPTp would result in 5\% of treated women having placental malaria. To detect a decrease in placental malaria from 20 to $5 \%$ with $66 \%$ of women in the 'two or more doses' strata compared to one or less doses of SP-IPTp and with a 95\% confidence level (CI) and a power of $0.8,116$ treated women and 60 untreated women were needed for a total of 176 women. To account for the design effect $(D E=2)$ of cluster sampling at the health facility level, this sample was doubled to 352 . Accounting for possible losses due to problems with specimen quality, $10 \%$ was added to this amount for a total of 387 patients.

\section{Statistical analysis}

Frequency counts and percentages were calculated for subject characteristics. The primary outcome was placental infection according to histopathology; secondary outcomes were LBW, preterm delivery, SGA, composite poor birth outcome, maternal anaemia, and any infection (defined as pathology class 1-4, OR blood smear positive (maternal, placental or cord blood)). The independent variable of interest was doses of SP-IPTp, evaluated for three categorical SP-IPTp variables: (i) $<$ two versus $\geq$ two SP-IPTp doses, with the $\geq$ two SP-IPTp doses representing full uptake of the WHO IPTp recommendations for subSaharan African countries at the time of the study; (ii) < three versus $\geq$ three SP-IPTp doses, with $\geq$ three SP-IPTp doses reflecting full uptake of the Zambian national policy at the time; and, (iii) two versus $\geq$ three SP-IPTp doses to assess the added benefit between two doses and three or more doses. All analyses were performed in SAS version 9.3 (SAS Institute, Inc, Cary, NC, USA) and PROC GENMOD was used for all Poisson regression models with robust standard errors [37]. Bivariate, unadjusted prevalence ratios for the SP-IPTp effects were calculated and multivariate Poisson regression models were fit to adjust for cofactors. Cofactors considered for the models were: age group $(<18,18-35,>35)$, gravidity, sleeping under an ITN the last night at home, living in a home treated with IRS, living in an urban area, facility of enrolment, delivering during the wet season (November-April), marital status, and taking SP-IPTp doses outside of antenatal clinic visits. Cofactors included in the final adjusted models were selected based on strength of association in bivariate models (at the 0.1 level of significance for any outcome), biological plausibility, model fit statistics, and the number of outcome events. To assess if SP-IPTp exhibited a dose-response trend, SP-IPTp was defined as a continuous variable in unadjusted and multivariate models. Finally, Pearson's Chi-square tests were employed to assess the effect of dose timing on outcomes. A twosided $\mathrm{p}$-value $<0.05$ was considered statistically significant.

\section{Human subjects review}

Ethical approval for this study was obtained from institutional review boards at both the US Centers for

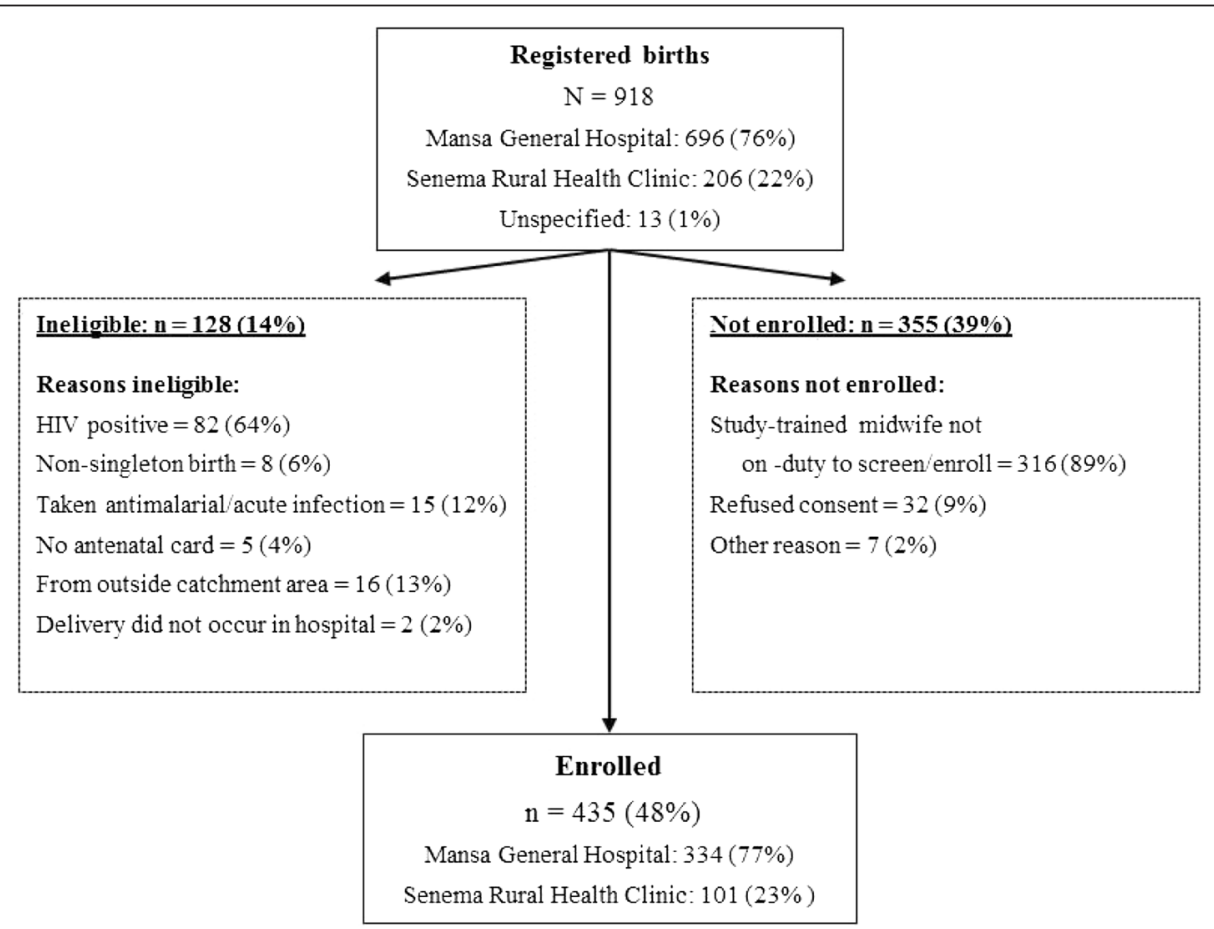

Figure 1 Flow chart of registered births and participant enrolment. 
Disease Control and Prevention and the Tropical Diseases Research Centre in Zambia. All participating women provided written informed consent.

\section{Results}

During the study period, a total of 918 births were registered at the two health facilities (Figure 1). Of these, 128 (14\%) births were screened and found ineligible. Reasons for ineligibility included HIV positivity, non-singleton birth, having taken an anti-malarial for acute malaria infection, no antenatal card available (e.i., undocumented HIV status), being from outside the Mansa District, and for not delivering in one of the two hospitals. In addition 355 (39\%) women were not enrolled because they refused consent, or there was no trained midwife on duty with sufficient time or study materials, or for other reasons. The most common reason that a birth was not screened for enrolment was because a trained midwife was not on duty, or was unable to conduct study procedures and still manage work priorities, 316 (89\%). While all births at the two facilities were documented in the routine registers, demographic information was not available for the women not screened because a survey-trained midwife was not on duty. Table 1 shows the characteristics of the 435 women enrolled into the study. The median age was 23 years with a range from 16-44 years; $42(10 \%)$ women were $<18$ years, and $33(8 \%)$ were $\geq 35$ years old. Thirty-seven per cent of women were pregnant for the first time (primigravid), and $51 \%$ were pregnant for the first or second time (paucigravid). Thirty-four (8\%) women did not take any SP-IPTp during pregnancy; 115 (26\%), 142 (33\%) and 144 (33\%) women took one, two or three + doses of SPIPTp, respectively. In all, 435 enrolled women took 840 doses of SP-IPTp. Of these, 46 (5\%) doses from 33 women were validated from self-report as being distinct from SP-IPTp doses recorded on the ANC card (13 additional doses were reported but could not be validated). Other subject characteristics are reported in Table 1.

Infant and maternal outcomes are reported in Table 2. Of infants born in the cohort, 8\% were LBW, 14\% were preterm and $18 \%$ were SGA. There were a total of 133 (31\%) infants born with a composite poor birth outcome (any of LBW, preterm or SGA). Of 31 LBW babies born alive, survivorship at six weeks was known for 28; 24 (86\%) were alive at six weeks after birth. There were a total of 15 infant deaths in the study: nine were stillbirths (eight $\geq 28$ weeks of gestation, one was $<28$ weeks of gestation), and six infants died immediately after, or within six weeks, of birth. No maternal deaths occurred among the cohort. At the time of delivery, more than one-third of all women had moderate anaemia, 157 (36\%) women, while 14 (3\%) women had severe anaemia.
Table 1 Subject characteristics

\begin{tabular}{ll}
\hline Characteristic & $\mathbf{n} / \mathbf{N}^{\mathbf{a}}$ (\%) \\
\hline Aged $<18$ years old & $42 / 432(10)$ \\
Aged $\geq 35$ years old & $33 / 432(8)$ \\
Doses of SP'-IPTp ${ }^{\mathrm{C}}$ taken & \\
0 doses & $34 / 435(8)$ \\
1 dose & $115 / 435(26)$ \\
2 doses & $142 / 435(33)$ \\
3 doses & $135 / 435(31)$ \\
4 doses & $9 / 435(2)$ \\
Primigravid women & $159 / 433(37)$ \\
Paucigravid women & $218 / 432(51)$ \\
Multigravid women & $214 / 432(50)$ \\
From rural communities & $255 / 433(59)$ \\
Married & $365 / 433(84)$ \\
Home is treated with indoor residual spraying & $149 / 411(35)$ \\
Owns an insecticide treated net (ITN) & $217 / 435(50)$ \\
Slept under ITN during the last night at home & $180 / 435(42)$ \\
Delivered during the wet season & $345 / 434(80)$ \\
Self-reported doses of SP-IPTp & $33 / 435(8)$
\end{tabular}

${ }^{\mathrm{a}}$ Total contributing responses.

${ }^{\mathrm{b}} \mathrm{SP}=$ sulphadoxine pyrimethamine.

${ }^{\mathrm{C}} \mathrm{PPT} \mathrm{P}=$ intermittent preventive treatment for malaria in pregnancy.

Table 2 Outcome frequencies

\begin{tabular}{|c|c|}
\hline Outcome & $\mathrm{n} / \mathrm{N}^{\mathrm{a}}(\%)$ \\
\hline \multicolumn{2}{|l|}{ Placental infection by histopathology } \\
\hline Abnormal (classes 1-4) & $162 / 435(37)$ \\
\hline Active infection (class 1) & $18 / 435(4)$ \\
\hline Chronic infection (classes 2 and 3) & $32 / 435(7)$ \\
\hline Past infection (class 4) & $112 / 435(26)$ \\
\hline Low birth weight (LBW) infant & $34 / 435(8)$ \\
\hline Preterm infant & $61 / 429(14)$ \\
\hline Small for gestational age (SGA) infant & $76 / 428(18)$ \\
\hline $\begin{array}{l}\text { Composite birth outcome (LBW, preterm, } \\
\text { or SGA) }\end{array}$ & $133 / 435(31)$ \\
\hline Stillbirth & $9 / 432(2)$ \\
\hline Infant death within six weeks of birth & $6 / 431(1)$ \\
\hline \multicolumn{2}{|l|}{ Maternal anaemia } \\
\hline Moderate anaemia $(\geq 8.0 \mathrm{Hb}<11.0 \mathrm{~g} / \mathrm{dl})$ & $157 / 435(36)$ \\
\hline Severe anaemia $(\mathrm{Hb}<8.0 \mathrm{~g} / \mathrm{dl})$ & $14 / 435(3)$ \\
\hline \multicolumn{2}{|l|}{ Blood smear positive for malaria parasites } \\
\hline Maternal peripheral & $21 / 419(5)$ \\
\hline Cord blood & $9 / 428(2)$ \\
\hline Placental & $19 / 430(4)$ \\
\hline Any smear & $28 / 420(7)$ \\
\hline Parasite density (parasites/ul); min, max, median & $106.7,>200,000,3333.3$ \\
\hline
\end{tabular}

${ }^{a}$ Number with outcome/total contributing responses. 
Blood smear analysis showed that $21(5 \%)$, nine (2\%), and 19 (4\%) had a positive P. falciparum maternal peripheral, cord and placental blood smear, respectively; no other malaria species were detected. Twenty-eight (7\%) subjects had any positive blood smear. More than one-third of all placental histopathology specimens were abnormal for malaria infection 162 (37\%), the majority of these being classified as past infection (class 4), 112 (26\%). Placental histopathology identified 18 (4\%) active infections (class 1), and $32(7 \%)$ chronic infections (classes 2 and 3).

An unadjusted analysis examining the effects of SP exposure dichotomized in various ways and its effects on the outcomes of interest is summarized in Table 3. Two or more SP-IPTp doses among all women was associated with a protective effect for placental infection, LBW, preterm delivery, composite poor birth outcome, and any infection. Three doses of SP-IPTp compared to $<$ three doses remained significantly associated with a reduction of LBW, preterm deliveries and composite poor birth outcome, for women of all gravidity. The difference between two and three or more SP-IPTp doses was not significantly different for any outcome or gravidity level.

Outcomes also varied by gravidity (Table 3 ). Paucigravid women were more likely to be associated with protection

Table 3 Unadjusted model: SP ${ }^{a}$-IPTp ${ }^{b}$ doses (categorical variables) associated with outcomes of interest

\begin{tabular}{|c|c|c|c|}
\hline Unadjusted model & $\begin{array}{l}\text { IPTp }<2 \text { vs } \geq 2 \text { SP doses } \\
\text { n, PR }\left(95 \% \mathrm{Cl}^{\mathrm{d}}\right)\end{array}$ & $\begin{array}{l}\text { IPTp }<3 \text { vs } \geq 3 \text { SP doses } \\
\mathrm{n}, \mathrm{PR}(95 \% \mathrm{Cl})\end{array}$ & $\begin{array}{l}\text { IPTp } 2 \text { vs } \geq 3 \text { SP doses } \\
\text { n, PR }(95 \% \mathrm{Cl})\end{array}$ \\
\hline \multicolumn{4}{|l|}{ Placental infection ${ }^{\mathrm{e}}$} \\
\hline All gravidity & $435,0.78(0.61-0.99)$ & $435,0.80(0.61-1.06)$ & $286,0.89(0.64-1.23)$ \\
\hline Paucigravid & $218,0.67(0.51-0.88)$ & $218,0.80(0.57-1.12)$ & $147,0.98(0.66-1.47)$ \\
\hline Multigravid & $214,0.93(0.60-1.44)$ & $214,0.81(0.50-1.30)$ & $137,0.79(0.46-1.36)$ \\
\hline \multicolumn{4}{|c|}{ Low birth weight (<2,500 g) } \\
\hline All gravidity & $435,0.41(0.22-0.79)$ & $435,0.35(0.14-0.88)$ & $286,0.49(0.17-1.41)$ \\
\hline Paucigravid & $218,0.27(0.09-0.77)$ & $218,0.36(0.08-1.56)$ & $147,0.75(0.13-4.38)$ \\
\hline Multigravid & $214,0.51(0.21-1.19)$ & $214,0.23(0.05-0.96)$ & $137,0.25(0.05-1.16)$ \\
\hline \multicolumn{4}{|l|}{ Preterm (<37 weeks) } \\
\hline All gravidity & $429,0.51(0.32-0.81)$ & $429,0.40(0.21-0.77)$ & $281,0.50(0.24-1.02)$ \\
\hline Paucigravid & $216,0.81(0.43-1.51)$ & $216,0.64(0.31-1.34)$ & $146,0.66(0.29-1.47)$ \\
\hline Multigravid & $210,0.26(0.12-0.56)$ & $210,0.16(0.04-0.67)$ & $133,0.29(0.06-1.39)$ \\
\hline \multicolumn{4}{|c|}{ Small for gestational age } \\
\hline All gravidity & $428,0.95(0.62-1.45)$ & $428,0.83(0.53-1.30)$ & $281,0.81(0.48-1.35)$ \\
\hline Paucigravid & 216, $1.11(0.62-1.99)$ & $216,0.94(0.53-1.69)$ & $146,0.88(0.46-1.67)$ \\
\hline Multigravid & $209,0.79(0.41-1.52)$ & $209,0.68(0.32-1.43)$ & $133,0.70(0.29-1.66)$ \\
\hline \multicolumn{4}{|c|}{ Composite birth outcome $e^{f}$} \\
\hline All gravidity & $435,0.74(0.56-0.98)$ & $435,0.64(0.45-0.90)$ & $286,0.69(0.47-1.01)$ \\
\hline Paucigravid & $218,0.99(0.67-1.45)$ & $218,0.82(0.55-1.25)$ & $147,0.79(0.50-1.25)$ \\
\hline Multigravid & $214,0.50(0.32-0.79)$ & $214,0.43(0.23-0.80)$ & $137,0.55(0.27-1.12)$ \\
\hline \multicolumn{4}{|l|}{ Anemia $(\mathrm{Hb}<11 \mathrm{~g} / \mathrm{dl})$} \\
\hline All gravidity & $434,0.89(0.70-1.13)$ & $434,0.87(0.67-1.13)$ & $285,0.90(0.67-1.22)$ \\
\hline Paucigravid & $217,0.75(0.55-1.03)$ & $217,0.72(0.49-1.06)$ & $146,0.79(0.51-1.22)$ \\
\hline Multigravid & $214,1.06(0.74-1.53)$ & $214,1.08(0.75-1.54)$ & $137,1.06(0.69-1.62)$ \\
\hline \multicolumn{4}{|l|}{ Any infection ${ }^{g}$} \\
\hline All gravidity & $435,0.78(0.61-0.99)$ & $435,0.79(0.60-1.04)$ & $286,0.87(0.64-1.20)$ \\
\hline Paucigravid & $218,0.66(0.50-0.86)$ & $218,0.80(0.57-1.10)$ & $147,0.99(0.67-1.46)$ \\
\hline Multigravid & $214,0.95(0.62-1.47)$ & $214,0.79(0.49-1.26)$ & $137,0.75(0.44-1.28)$ \\
\hline
\end{tabular}

${ }^{\mathrm{a}} \mathrm{SP}=$ sulphadoxine pyrimethamine.

${ }^{\mathrm{b}} \mathrm{PPTp}=$ intermittent preventive treatment for malaria in pregnancy.

${ }^{\mathrm{C}} \mathrm{PR}=$ prevalence ratio.

${ }^{d}$ Denotes $95 \%$ confidence interval.

eplacental infection outcome: classes 1-4 vs class 5.

${ }^{f}$ Composite birth outcome defined as infants born with any of: LBW, SGA, or preterm.

${ }^{9}$ Any infection defined as: pathology classes 1-4, blood smear positive (maternal, placental or cord blood). 
from LBW, placental histopathology malaria abnormalities, or any infection. In contrast, multigravid women were more likely to be protected from preterm delivery, and composite poor birth outcome. In addition, among multigravid women, protection from LBW was not associated with two doses of SP-IPTp, but was observed with three or more SP-IPTp doses.

Outcomes significant in the unadjusted analysis were assessed with Poisson multivariate regression models, including gravidity as an interaction term with SP-IPTp, and age group $(<18,18-35,>35)$, sleeping under an ITN the previous night at home, living in a home that is IRS treated, living in an urban area, facility of enrolment, delivering during the wet season (November-April), and marital status (Table 4). Self-reported SP-IPTp doses were considered and tested as a cofactor, but were not found to be significant (at a 0.1 level) for any outcome of interest. All three SP-IPTp dosing comparisons were assessed, although for some outcomes, SP-IPTp two versus $\geq$ three doses could not be computed because of an inadequate number of responses. In paucigravid women two or more SP-IPTp doses were protective for LBW and any infection. Among multigravid women, $\geq$ two and $\geq$ three SP-IPTp doses were associated with fewer preterm deliveries and composite poor birth outcomes. Three or more doses of SP-IPTp compared to two doses were not significant for either of the outcomes for which it could be computed (placental infection, and any infection).

Table 5 summarizes the results of an unadjusted assessment of the dose-response effect of SP-IPTp on outcomes, considering SP-doses as a continuous variable, and the interaction of gravidity and number of SP-IPTp doses taken into account. Among all women, the trend p-values was significant $(<0.05)$ for outcomes of placental infection by histopathology, LBW, preterm delivery, composite poor birth outcome, and any infection.

Multivariate Poisson regression analysis with the same covariates as previously mentioned (age group $(<18$, $18-35,>35)$, sleeping under an ITN the previous night at home, living in a home that is IRS treated, living in an urban area, facility of enrolment, delivering during the wet season, and marital status) shows that protection from LBW was significantly associated with increased SP-IPTp doses for both paucigravid and multigravid women (Table 6). For each dose of SP-IPTp there was a 46 and $37 \%$ decrease in the frequency of LBW among paucigravid and multigravid women, respectively. Placental infection by histopathology and any infection were not significantly associated with SP-IPTp doses in this analysis. Among multigravid women, the dose-response effect from SPIPTp remained significantly associated with protection from preterm delivery and the composite poor birth outcome.

Table 4 Multivariate regression: SP $^{a}$-IPTp ${ }^{b}$ doses (categorical variables) associated with outcomes of interest

\begin{tabular}{|c|c|c|c|}
\hline Adjusted model ${ }^{c}$ & $<2$ vs $\geq 2$ doses $\mathrm{PR}^{\mathrm{d}}\left(95 \% \mathrm{Cl}^{\mathrm{e}}\right)$ & $<3$ vs $\geq 3$ doses $\mathrm{PR}(95 \% \mathrm{Cl})$ & 2 vs $\geq 3$ doses $\mathrm{PR}(95 \% \mathrm{Cl})$ \\
\hline \multicolumn{4}{|l|}{ Placental infection ${ }^{f}$} \\
\hline Paucigravid & $0.77(0.58-1.01)$ & $0.89(0.64-1.23)$ & $1.06(0.71-1.57)$ \\
\hline Multigravid & $1.00(0.66-1.52)$ & $0.87(0.55-1.39)$ & $0.83(0.49-1.39)$ \\
\hline \multicolumn{4}{|c|}{ Low birth weight $(<2,500 \mathrm{~g})$} \\
\hline Paucigravid & $0.33(0.12-0.91)$ & $0.43(0.10-1.81)$ & Could not compute \\
\hline Multigravid & $0.51(0.21-1.25)$ & $0.26(0.06-1.11)$ & Could not compute \\
\hline \multicolumn{4}{|l|}{ Preterm (<37 weeks) } \\
\hline Paucigravid & $0.93(0.49-1.78)$ & $0.70(0.34-1.44)$ & Could not compute \\
\hline Multigravid & $0.28(0.13-0.60)$ & $0.15(0.04-0.55)$ & Could not compute \\
\hline \multicolumn{4}{|c|}{ Composite birth outcome ${ }^{g}$} \\
\hline Paucigravid & $1.09(0.73-1.61)$ & $0.84(0.54-1.31)$ & Could not compute \\
\hline Multigravid & $0.50(0.32-0.78)$ & $0.43(0.24-0.80)$ & Could not compute \\
\hline \multicolumn{4}{|l|}{ Any infection ${ }^{h}$} \\
\hline Paucigravid & $0.76(0.58-0.99)$ & $0.88(0.65-1.21)$ & $1.06(0.72-1.55)$ \\
\hline Multigravid & $1.04(0.69-1.58)$ & $0.86(0.54-1.36)$ & $0.79(0.48-1.33)$ \\
\hline
\end{tabular}

${ }^{\mathrm{a}} \mathrm{SP}=$ sulphadoxine pyrimethamine.

${ }^{\mathrm{b}} \mathrm{PPTp}=$ intermittent preventive treatment for malaria in pregnancy.

${ }^{c}$ Model adjusted for: Age group $(<18,18-35,>35)$, gravidity as an interaction term, sleeping under an ITN the last night at home, living in a home treated with

IRS, urban, facility, delivered during the wet season, marital status.

${ }^{\mathrm{d}} \mathrm{PR}=$ prevalence ratio.

${ }^{\mathrm{e}} \mathrm{Cl}=95 \%$ confidence interval.

fPlacental infection outcome: histopathology classes 1-4 vs class 5.

${ }^{9}$ Composite birth outcome defined as infants born with any of: LBW, SGA or preterm.

${ }^{h}$ Any infection defined as: pathology classes 1-4, blood smear positive (maternal, placental or cord blood). 
Table 5 Unadjusted trend analysis: SPa - IPTp ${ }^{\mathrm{b}}$ doses (continuous variable) associated with outcomes of interest

\begin{tabular}{lll}
\hline Outcome & $\begin{array}{l}\text { Trend prevalence ratio } \\
\text { estimate }\left(95 \% \mathrm{Cl}^{\mathrm{c}}\right)\end{array}$ & $\begin{array}{l}\text { Trend } \\
\text { p-value }\end{array}$ \\
\hline
\end{tabular}

Placental infection $^{d}$

All gravidity

$0.87(0.77-0.99)$

Paucigravid

$0.84(0.72-0.97)$

0.03

Multigravid

$0.91(0.73-1.12)$

0.37

Low birth weight $(<2,500 \mathrm{~g})$

$$
\text { All gravidity }
$$

$0.59(0.43-0.81)$

Paucigravid

$0.50(0.29-0.85)$

Multigravid

$0.62(0.42-0.90)$

Preterm (<37 weeks)

$$
\text { All gravidity }
$$

$0.65(0.51-0.81)$

Paucigravid

$0.82(0.60-1.12)$

$0.47(0.33-0.66)$

Multigravid

$0.93(0.75-1.15)$

0.99 (0.74-1.32)

$0.85(0.62-1.17)$

$<0.01$

0.01

0.01

Small for gestational age

All gravidity

Paucigravid

Multigravid

Composite birth outcome

$$
\text { All gravidity }
$$

$0.80(0.70-0.92)$

$0.92(0.76-1.11)$

$0.67(0.54-0.84)$

$<0.01$

0.21

$<0.01$

Paucigravid

Multigravid

$0.93(0.82-1.05)$

$0.84(0.72-0.99)$

$1.03(0.86-1.23)$

0.49

0.94

0.32

Anemia $(\mathrm{Hb}<11 \mathrm{~g} / \mathrm{dl})$

All gravidity

Paucigravid

Multigravid

$0.87(0.77-0.98)$

$0.84(0.72-0.96)$

0.03

All gravidity

$0.91(0.74-1.12)$

0.01

Multigravid

${ }^{a} \mathrm{SP}=$ sulphadoxine pyrimethamine

$\mathrm{b}_{\text {IPTp }}=$ intermittent preventive treatment for malaria in pregnancy.

${ }^{\mathrm{C}} \mathrm{Cl}=95 \%$ confidence interval

dPlacental infection outcome: histopathology classes 1-4 vs class 5 .

${ }^{\text {e}}$ Composite birth outcome defined as infants born with any of: LBW, SGA,

or preterm.

${ }^{\mathrm{f}}$ Any infection defined as pathology classes 1-4, blood smear positive (maternal, placental or cord blood).

The effect of the timing of the SP-IPTp doses on outcomes was assessed among 392 women who had taken $\geq$ one SP-IPTp dose, and for which the complete date of the SP-IPTp dose was known. A total of 172 (44\%) women took their last dose of SP-IPTp within the four weeks prior to birth. The only outcome that was significantly associated with the timing of the last SP-IPTp dose (Pearson's Chi-square test) was parasite positivity (parasites found on any blood smear, or histopathology classes $1-3) ; 16.8 \%$ of women were parasite positive among those who did not take SP-IPTp during
Table 6 Multivariate logistic regression trend analysis:

\begin{tabular}{|c|c|c|}
\hline Adjusted model $^{c}$ & $\begin{array}{l}\text { Trend prevalence ratio } \\
\text { estimate }\left(95 \% \mathrm{Cl}^{\mathrm{d}}\right)\end{array}$ & $\begin{array}{l}\text { Trend } \\
\text { p-value }\end{array}$ \\
\hline \multicolumn{3}{|l|}{ Placental infection ${ }^{e}$} \\
\hline Paucigravid & $0.88(0.77-1.04)$ & 0.13 \\
\hline Multigravid & $0.94(0.76-1.16)$ & 0.58 \\
\hline \multicolumn{3}{|c|}{ Low birth weight $(<2,500 \mathrm{~g})$} \\
\hline Paucigravid & $0.54(0.33-0.90)$ & 0.02 \\
\hline Multigravid & $0.63(0.41-0.97)$ & 0.04 \\
\hline \multicolumn{3}{|c|}{ Preterm (<37 weeks) } \\
\hline Paucigravid & $0.85(0.62-1.17)$ & 0.32 \\
\hline Multigravid & $0.48(0.34-0.69)$ & $<0.0001$ \\
\hline \multicolumn{3}{|c|}{ Composite birth outcome $^{f}$} \\
\hline Paucigravid & $0.94(0.77-1.15)$ & 0.56 \\
\hline Multigravid & $0.66(0.53-0.82)$ & $<0.001$ \\
\hline \multicolumn{3}{|l|}{ Any infection ${ }^{9}$} \\
\hline Paucigravid & $0.89(0.77-1.03)$ & 0.13 \\
\hline Multigravid & $0.95(0.77-1.17)$ & 0.63 \\
\hline
\end{tabular}
SP $^{a}$-IPTp ${ }^{b}$ doses (continuous variable) associated with outcomes of interest

${ }^{a} \mathrm{SP}=$ sulphadoxine pyrimethamine.

$\mathrm{b}_{\text {IPTp }}=$ intermittent preventive treatment for malaria in pregnancy.

${ }^{c}$ Model adjusts for: Age group $(<18,18-35,>35)$, gravidity as an interaction term, sleeping under an ITN the last night at home, living in a home treated with IRS, urban, facility, delivered during the wet season, marital status. ${ }^{d} \mathrm{Cl}=95 \%$ confidence interval.

ePlacental infection outcome: histopathology classes 1-4 vs class 5 .

${ }^{f}$ Composite birth outcome defined as infants born with any of: LBW, SGA, or preterm.

${ }^{9}$ Any infection defined as pathology classes $1-4$, blood smear positive (maternal, placental or cord blood).

the four weeks prior to birth versus $8.7 \%$ positive among those who did take SP-IPTp during the four weeks prior to birth $(\mathrm{p}=0.02)$. There was no effect of the timing of SP-IPTp on the following outcomes: placental infection, moderate anaemia, LBW, pre-term delivery, SGA, composite poor birth outcome, and any infection.

\section{Discussion}

This study found that SP-IPTp in Mansa, Zambia protected pregnant women from poor birth outcomes, and that the degree and type of protection afforded by SPIPTp was dependent on the specific outcome, gravidity, and number of SP-IPTp doses taken. Two or more doses of SP-IPTp were determined to be a threshold associated with protection of paucigravid women from outcomes of placental malaria, LBW, and any malaria infection. Likewise, at least two or more doses of SP-IPTp were associated with protection of multigravid women from preterm delivery and composite poor birth outcome. In addition, analysis of SP-IPTp as a continuous variable, to assess the trend-effect for each subsequent dose, showed that each dose of SP-IPTp confers a protective benefit for LBW 
among women of any gravidity (Table 6). Furthermore, the setting in which these outcomes were observed was described in an SP-IPTp therapeutic efficacy study, conducted in parallel to this one, in Mansa, Zambia, showing that among asymptomatic, parasitaemic, HIV-negative women enrolled in their second or third trimester, SPIPTp had a $26 \%$ failure rate in terms of recurrent parasitaemia, and found an intermediate prevalence of genetic mutations that contribute to SP resistance [25].

This work provides valuable information on the continued effectiveness of SP-IPTp in transmission settings that have moderate prevalence of dhps and $d h f r$ mutant alleles. Previous studies have examined SP-IPTp outcomes in areas of high prevalence of the quintuple SP mutations [22,38-42], sometimes demonstrating that protective effectiveness is waning or SP-IPTp is harmful [22,43]. Alternatively, in the West Africa region, while SP mutations are not uncommon, there is lower prevalence of the quintuple mutation $[40,44]$ and continued effectiveness of SP-IPTp is likely to remain high $[45,46]$.

In 2012 WHO clarified SP-IPTp recommendations for areas of moderate-to high transmission, continuing the recommendation that women should receive four antenatal care visits spaced at least one month apart in the second and third trimester, but specifying that a dose of SP-IPTp should be administered at each visit [7]; Zambia adopted a policy aligned with the current WHO recommendations. Results presented herein support the current SP-IPTp policies and provide further evidence that pregnant women continue to benefit from three or more doses of SP-IPTp, by showing that SP-IPTp had a protective dose-response effect among all women for the outcome of LBW (Table 6). Operationally, the clarified guidelines should be straightforward for antenatal clinics to implement, increasing the likelihood that women will benefit from an increased number of SP-IPTp doses. To improve SP-IPTp uptake, measures such as supervision of healthcare workers to improve provision of SP-IPTp, improvement of supply chain management to prevent SP stock-outs, and text messages serving as antenatal care reminders, could be considered.

Results herein illustrate differences in consideration of outcomes and gravidity. For the primary outcome of placental infection, at least two doses of SP-IPTp are protective for paucigravid women and not multigravid women. In addition, paucigravid women were similarly protected from LBW and any infection, in contrast to multigravid women. The outcomes where SP-IPTp is significantly protective only among multigravid women are preterm delivery and the composite poor birth outcome (which includes, and is driven by preterm delivery) (Table 4). While there is not a single compelling reason to explain this gravidity difference for the preterm outcome, the following explanations are plausible: (i) among primigravid women, it is possible that there was not sufficient power to detect a protective effect against preterm delivery, (ii) preterm is assessed using last menstrual period and Ballard scores, methodologies which can be subject to error, and could have resulted in misclassification bias. These results support the conclusion that SP-IPTp confers a protective effect among women of any gravidity status, although the protection is stronger among paucigravid women for the outcomes of LBW, placental infection, and any infection.

This study has the following limitations: it was designed to be observational where women were not randomized to receive different amounts of SP-IPTp doses. While relatively equal distribution of SP-IPTp for one, two or three or more doses $(115,142$, and 144 women, respectively) was observed, only 34 women received zero doses of SP-IPTp, limiting the ability to analyse the effectiveness of any SP-IPTp doses compared to the group of women who received none. It is possible that there is misclassification of some SP-IPTp doses, especially for those 46 doses not recorded on ANC cards. Treating SP-IPTp dosage as a continuous variable in modelling may mask potential non-linear effects. It was not possible to determine the quality of the SP doses provided at antenatal clinics across the Mansa District area. However, the dose-timing analysis found that taking SP-IPTp in the four weeks prior to birth was associated with a decrease in parasite positivity, suggesting generally that the administered SP had antiparasitic properties. One-third of all births registered during the study period at the two facilities were not screened for enrolment, and since demographic information was not available for the non-screened women it is impossible to evaluate whether women enrolled were representative of the true population of women giving birth at the two sites. Although all enrolled women had an HIV-negative test result recorded during their pregnancy, it is possible that HIV-positive women were enrolled; however the effect of this error would have biased results towards the null hypothesis. Additionally, it was not possible to control for antenatal visits, and this could affect the study outcomes, including birth weight [47].

\section{Conclusions}

In Mansa, Zambia, an area of moderate SP resistance, two or more doses of SP-IPTp are associated with a protective effect from malaria in pregnancy, especially among paucigravid women. Each dose of SP-IPTp contributed to a 46 and 37\% decrease in the frequency of LBW among paucigravid and multigravid women, respectively. The results herein suggest that SP-IPTp confers protective benefits to pregnant women and their infants in this transmission setting, despite a previously described intermediate prevalence of genetic mutations that contribute to SP resistance; the SP-IPTp strategy should continue to be supported. To protect women from deleterious effects of 
malaria during pregnancy, update of SP-IPTp should be improved; SP supply chain management, implementing text message antenatal reminders, and supervision of healthcare providers could be considered to ensure women have optimal access to SP-IPTp.

\section{Competing interests}

The authors declared that they have no competing interests.

\section{Author's contributions}

KEM led study implementation, provided technical expertise, conducted statistical analysis, and drafted the manuscript. VC supervised logistics for study implementation and reviewed manuscript. BLK served as the study coordinator, implemented the study, and reviewed the manuscript. MN was the key point person for laboratory quality control and laboratory data. LM and $\mathrm{CM}$ conducted the expert pathology analysis and reviewed the manuscript. REW assisted with statistical analysis and reviewed the manuscript. SJF provided technical expertise for the design of the study and reviewed the manuscript. MK provided technical expertise and reviewed the manuscript. ASC provided technical expertise, supervised implementation of the study, and reviewed the manuscript. KRT conceived of and designed the study, wrote the protocol, helped with implementation, provided technical expertise, and assisted with drafting the manuscript. All authors read and approved the final manuscript.

\section{Acknowledgements}

We gratefully acknowledge the participation of the patients, midwives, hospital administrators in this study. Julie Gutman, Feiko ter Kuile and the MiP Consortium provided technical guidance which improved the study implementation and analysis. This report was made possible through support provided by the President's Malaria Initiative, US Agency for International Development, under the terms of an Interagency Agreement with the Centers for Disease Control and Prevention. The findings, results and opinions expressed herein are those of the authors and do not necessarily reflect the views of the US Agency for International Development or the Centers for Disease Control and Prevention.

\section{Author details}

Malaria Branch, Division of Parasitic Diseases and Malaria, Center for Global Health, Centers for Disease Control and Prevention, Atlanta, GA, USA. ${ }^{2}$ School of Medicine, Department of Public Health, University of Zambia, Lusaka, Zambia. ${ }^{3}$ United States Peace Corps, Lusaka, Zambia. ${ }^{4}$ Tropical Diseases Research Centre, Ndola, Zambia. ${ }^{5}$ Ndola Central Hospital, Ndola, Zambia. ${ }^{6}$ Wusakile Mine Hospital, Kitwe, Zambia. ${ }^{7}$ National Malaria Control Centre, Lusaka, Zambia.

Received: 9 October 2014 Accepted: 19 January 2015

Published online: 07 February 2015

\section{References}

1. Dellicour S, Tatem AJ, Guerra CA, Snow RW, ter Kuile FO. Quantifying the number of pregnancies at risk of malaria in 2007: a demographic study. PLoS Med. 2010;7:e1000221.

2. Central Statistical Office (CSO) MoHM, Tropical Diseases Research Centre (TDRC), University of Zambia aMII. Zambia Demographic and Health Survey 2007. Calverton, Maryland, USA: CSO and Macro International Inc; 2009.

3. Desai M, ter Kuile FO, Nosten F, McGready R, Asamoa K, Brabin B, et al. Epidemiology and burden of malaria in pregnancy. Lancet Infect Dis. 2007;7:93-104

4. Steketee RW, Nahlen BL, Parise ME, Menendez C. The burden of malaria in pregnancy in malaria-endemic areas. Am J Trop Med Hyg. 2001;64:28-35.

5. Ministry of Health. Zambia national malaria indicator survey 2010 . Government of the Republic of Zambia; 2010

6. Ministry of Health. A 6-year strategic plan: A road map for impact on malaria in Zambia, 2006-2011. Government of the Republic of Zambia.

7. WHO. Intermittent preventive treatment of malaria in pregnancy using sulfadoxine-pyrimethamine (IPTp-SP). Updated WHO Policy Recommendation (October 2012). [http://www.who.int/malaria/iptp_sp_updated_policy_ recommendation_en_102012.pdf]
8. Peters PJ, Thigpen MC, Parise ME, Newman RD. Safety and toxicity of sulfadoxine/pyrimethamine: implications for malaria prevention in pregnancy using intermittent preventive treatment. Drug Saf. 2007;30:481-501.

9. Schultz L, Steketee RW, Macheso A, Kazembe P, Chitsulo L, Wirima JJ. The efficacy of antimalarial regimens containing sulfadoxine-pyrimethamine and/or chloroquine in preventing peripheral and placental Plasmodium falciparum infection among pregnant women in Malawi. Am J Trop Med Hyg. 1994:51:515-22.

10. Parise ME, Ayisi JG, Nahlen BL, Schultz LJ, Roberts JM, Misore A, et al. Efficacy of sulfadoxine-pyrimethamine for prevention of placental malaria in an area of Kenya with a high prevalence of malaria and human immunodeficiency virus infection. Am J Trop Med Hyg. 1998;59:813-22.

11. Shulman CE, Dorman EK, Cutts F, Kawuondo K, Bulmer JN, Peshu N, et al. Intermittent sulphadoxine-pyrimethamine to prevent severe anaemia secondary to malaria in pregnancy: a randomised placebo-controlled trial. Lancet. 1999;353:632-6.

12. Kayentao K, Kodio M, Newman RD, Maiga H, Doumtabe D, Ongoiba A, et al. Comparison of intermittent preventive treatment with chemoprophylaxis for the prevention of malaria during pregnancy in Mali. J Infect Dis. 2005;191:109-16

13. Challis K, Osman NB, Cotiro M, Nordahl G, Dgedge M, Bergstrom S. Impact of a double dose of sulphadoxine-pyrimethamine to reduce prevalence of pregnancy malaria in southern Mozambique. Trop Med Int Health. 2004;9:1066-73

14. Filler SJ, Kazembe P, Thigpen M, Macheso A, Parise ME, Newman RD, et al. Randomized trial of 2-dose versus monthly sulfadoxine-pyrimethamine intermittent preventive treatment for malaria in HIV-positive and HIV-negative pregnant women in Malawi. J Infect Dis. 2006;194:286-93.

15. Rogerson SJ, Chaluluka E, Kanjala M, Mkundika P, Mhango C, Molyneux ME. Intermittent sulfadoxine-pyrimethamine in pregnancy: effectiveness against malaria morbidity in Blantyre, Malawi, in 1997-99. Trans R Soc Trop Med Hyg. 2000;94:549-53.

16. van Eijk AM, Ayisi JG, ter Kuile FO, Otieno JA, Misore AO, Odondi JO, et al. Effectiveness of intermittent preventive treatment with sulphadoxinepyrimethamine for control of malaria in pregnancy in western Kenya: a hospital-based study. Trop Med Int Health. 2004;9:351-60.

17. Sirima SB, Cotte AH, Konate A, Moran AC, Asamoa K, Bougouma EC, et al Malaria prevention during pregnancy: assessing the disease burden one year after implementing a program of intermittent preventive treatment in Koupela District, Burkina Faso. Am J Trop Med Hyg. 2006;75:205-11.

18. Njagi JK, Magnussen P, Estambale B, Ouma J, Mugo B. Prevention of anaemia in pregnancy using insecticide-treated bednets and sulfadoxinepyrimethamine in a highly malarious area of Kenya: a randomized controlled trial. Trans R Soc Trop Med Hyg. 2003;97:277-82.

19. Verhoeff FH, Brabin BJ, Chimsuku L, Kazembe P, Russell WB, Broadhead RL. An evaluation of the effects of intermittent sulfadoxine-pyrimethamine treatment in pregnancy on parasite clearance and risk of low birthweight in rural Malawi. Ann Trop Med Parasitol. 1998;92:141-50.

20. Mharakurwa S, Kumwenda T, Mkulama MA, Musapa M, Chishimba S, Shiff $\mathrm{CJ}$, et al. Malaria antifolate resistance with contrasting Plasmodium falciparum dihydrofolate reductase (DHFR) polymorphisms in humans and Anopheles mosquitoes. Proc Natl Acad Sci U S A. 2011;108:18796-801.

21. Hamainza B, Chanda-kapata P, Moonga H, Chizema-Kawesha E. Therapeutic efficacy of artemether lumefantrine and sulphadoxine-pyrimethamine for treatment of uncomplicated Plasmodium falciparum Malaria in Zambian children under five years. Medical J Zambia. 2007;34:5.

22. Harrington WE, Mutabingwa TK, Kabyemela E, Fried M, Duffy PE. Intermittent treatment to prevent pregnancy malaria does not confer benefit in an area of widespread drug resistance. Clin Infect Dis. 2011;53:224-30.

23. Kalilani L, Mofolo I, Chaponda M, Rogerson SJ, Meshnick SR. The effect of timing and frequency of Plasmodium falciparum infection during pregnancy on the risk of low birth weight and maternal anemia. Trans R Soc Trop Med Hyg. 2010;104:416-22.

24. Kublin JG, Dzinjalamala FK, Kamwendo DD, Malkin EM, Cortese JF, Martino LM, et al. Molecular markers for failure of sulfadoxine-pyrimethamine and chlorproguanil-dapsone treatment of Plasmodium falciparum malaria. J Infect Dis. 2002;185:380-8

25. Tan KR, Katalenich BL, Mace KE, Nambozi M, Taylor SM, Meshnick SR, et al. Efficacy of sulphadoxine-pyrimethamine for intermittent preventive treatment of malaria in pregnancy, Mansa, Zambia. Malar J. 2014;13:227. 
26. Hamer DH, Mwanakasale V, Macleod WB, Chalwe V, Mukwamataba D, Champo D, et al. Two-dose versus monthly intermittent preventive treatment of malaria with sulfadoxine-pyrimethamine in HIV-seropositive pregnant Zambian women. J Infect Dis. 2007:196:1585-94.

27. Gutman J, Mwandama D, Wiegand RE, Ali D, Mathanga DP, Skarbinski J. Effectiveness of intermittent preventive treatment with sulfadoxinepyrimethamine during pregnancy on maternal and birth outcomes in Machinga district, Malawi. J Infect Dis. 2013;208:907-16.

28. Arinaitwe E, Ades V, Walakira A, Ninsiima B, Mugagga O, Patil TS, et al. Intermittent preventive therapy with sulfadoxine-pyrimethamine for malaria in pregnancy: a cross-sectional study from Tororo, Uganda. PLoS One. 2013;8:e73073.

29. Coulibaly SO, Kayentao K, Taylor S, Guirou EA, Khairallah C, Guindo N, et al. Parasite clearance following treatment with sulphadoxine-pyrimethamine for intermittent preventive treatment in Burkina-Faso and Mali: 42-day in vivo follow-up study. Malar J. 2014;13:41.

30. Iriemenam NC SM, Gatei W, van Eijk AM, Ayisi J, Kariuki S, Vanden Eng J, et al. Temporal trends of sulphadoxine-pyrimethamine (SP) drug-resistance molecular markers in Plasmodium falciparum parasites from pregnant women in western Kenya. Malaria J. 2012;11:134.

31. Ministry of Health. List of health facilities in Zambia. Lusaka, Zambia: Government of the Republic of Zambia; 2010.

32. Ballard JL, Khoury JC, Wedig K, Wang L, Eilers-Walsman BL, Lipp R. New Ballard Score, expanded to include extremely premature infants. J Pediatr. 1991;119:417-23.

33. Rogerson SJ, Pollina E, Getachew A, Tadesse E, Lema VM, Molyneux ME. Placental monocyte infiltrates in response to Plasmodium falciparum malaria infection and their association with adverse pregnancy outcomes. Am J Trop Med Hyg. 2003;68:115-9.

34. Landis SH, Ananth CV, Lokomba V, Hartmann KE, Thorp Jr JM, Horton A, et al. Ultrasound-derived fetal size nomogram for a sub-Saharan African population: a longitudinal study. Ultrasound Obstet Gynecol. 2009;34:379-86.

35. WHO. Basic Malaria Microscopy, Part 1. Second Edition editionth ed. Geneva, Switzerland: World Health Organization; 2010.

36. Ministry of Health. Zambia national malaria indicator survey 2008. Government of the Republic of Zambia; 2008.

37. Zou G. A modified poisson regression approach to prospective studies with binary data. Am J Epidemiol. 2004;159:702-6.

38. Taylor SM, Antonia AL, Chaluluka E, Mwapasa V, Feng G, Molyneux ME, et al. Antenatal receipt of sulfadoxine-pyrimethamine does not exacerbate pregnancy-associated malaria despite the expansion of drug-resistant Plasmodium falciparum: clinical outcomes from the QuEERPAM study. Clin Infect Dis. 2012;55:42-50.

39. Duah NO, Quashie NB, Abuaku BK, Sebeny PJ, Kronmann KC, Koram KA. Surveillance of molecular markers of Plasmodium falciparum resistance to sulphadoxine-pyrimethamine 5 years after the change of malaria treatment policy in Ghana. Am J Trop Med Hyg. 2012;87:996-1003.

40. Bertin G, Briand V, Bonaventure D, Carrieu A, Massougbodji A, Cot M, et al. Molecular markers of resistance to sulphadoxine-pyrimethamine during intermittent preventive treatment of pregnant women in Benin. Malar J. 2011;10:196.

41. Moussiliou A, Sissinto-Savi De Tove Y, Doritchamou J, Luty AJ, Massougbodji A, Alifrangis $\mathrm{M}$, et al. High rates of parasite recrudescence following intermittent preventive treatment with sulphadoxine-pyrimethamine during pregnancy in Benin. Malar J. 2013;12:195.

42. Taylor SM, Antonia A, Feng G, Mwapasa V, Chaluluka E, Molyneux M, et al. Adaptive evolution and fixation of drug-resistant Plasmodium falciparum genotypes in pregnancy-associated malaria: 9-year results from the QuEERPAM study. Infect Genet Evol. 2012;12:282-90.

43. Harrington WE, Mutabingwa TK, Muehlenbachs A, Sorensen B, Bolla MC, Fried $M$, et al. Competitive facilitation of drug-resistant Plasmodium falciparum malaria parasites in pregnant women who receive preventive treatment. Proc Natl Acad Sci U S A. 2009;106:9027-32.

44. Mockenhaupt FP, Bedu-Addo G, Eggelte TA, Hommerich L, Holmberg V, von

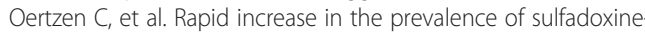
pyrimethamine resistance among Plasmodium falciparum isolated from pregnant women in Ghana. J Infect Dis. 2008;198:1545-9.

45. Diakite OS, Kayentao K, Traoré BT, Djimde A, Traore B, Diallo M, et al. Superiority of 3 over 2 doses of intermittent preventive treatment with sulfadoxine-pyrimethamine for the prevention of malaria during pregnancy in Mali: a randomized controlled trial. Clin Infect Dis. 2011:53:215-23.
46. Clerk CA, Bruce J, Affipunguh PK, Mensah N, Hodgson A, Greenwood B, et al. A randomized, controlled trial of intermittent preventive treatment with sulfadoxine-pyrimethamine, amodiaquine, or the combination in pregnant women in Ghana. J Infect Dis. 2008;198:1202-11.

47. Metgud CS, Naik VA, Mallapur MD. Factors affecting birth weight of a newborn-a community based study in rural Karnataka, India. PLoS One. 2012;7:e40040

\section{Submit your next manuscript to BioMed Central and take full advantage of:}

- Convenient online submission

- Thorough peer review

- No space constraints or color figure charges

- Immediate publication on acceptance

- Inclusion in PubMed, CAS, Scopus and Google Scholar

- Research which is freely available for redistribution 